\title{
Surfaces
}

\section{Vladimir Propp, Problemy komizma i smekha. Moskva:}

Labirint, 1999

\section{Éric Lozowy}

Volume 9, 2001

URI : https://id.erudit.org/iderudit/1065070ar

DOI : https://doi.org/10.7202/1065070ar

Aller au sommaire du numéro

Éditeur(s)

Les Presses de l’Université de Montréal

ISSN

1188-2492 (imprimé)

1200-5320 (numérique)

Découvrir la revue

Citer ce compte rendu

Lozowy, É. (2001). Compte rendu de [Vladimir Propp, Problemy komizma i

smekha. Moskva: Labirint, 1999]. Surfaces, 9. https://doi.org/10.7202/1065070ar d'utilisation que vous pouvez consulter en ligne.

https://apropos.erudit.org/fr/usagers/politique-dutilisation/ 


\section{Vladimir Propp - Problemy komizma i smekha}

Éric Lozowy

Russian and Slavic Studies Department

McGill University

Surfaces vol IX 1.02 (v.1.0F - 15/12/2001) - ISSN:

1188-2492

Tout texte reste la propriété de son auteur. Néanmoins, SURFACES demande d'être citée à l'occasion de toute autre publication du texte en question.

\section{Vladimir Propp, Problemy komizma i smekha. Moskva: Labirint, 1999.}

Le folkloriste russe Vladimir Propp (1895-1970) a connu une certaine notoriété dans les études littéraires en France et ailleurs en Occident durant les années 1960-1970, lorsque le formalisme et le structuralisme étaient des courants de pensée à la mode. Plusieurs de ses ouvrages ont été traduits en français, notamment la Morphologie du conte, son ouvrage le plus célèbre, qui a inspiré des théoriciens tels Bremond, Greimas et Todorov dans leur recherches sur les mécanismes narratifs. [ $\underline{\mathbf{1}}$ ]

L'oeuvre de Propp est essentiellement consacrée à l'étude du folklore. C'est, sans doute, en tant que folkloriste que l'auteur de la Morphologie du conte passera à la postérité. Néanmoins, durant les dernières années de sa vie, Propp avait commencé à explorer un autre domaine de recherche en rédigeant un ouvrage intitulé Problemy komizma i smekha (Problèmes du comique et du rire). Il imitait ainsi d'autres grands théoriciens du vingtième siècle, tels Bakhtine, Bergson et Freud, qui s'étaient intéressés à la question du rire. 
L'ouvrage est resté inachevé et n'a été publié que de façon posthume, en 1976, dans une version lacunaire truffée d'erreurs. Cette publication a eu peu de résonnance, autant en Russie qu'en Occident. Un quart de siècle plus tard, la maison d'édition moscovite Labirint, qui a entrepris la publication des oeuvres complètes de Propp, nous propose une nouvelle version des Problèmes du comique et du rire, plus soignée, avec un commentaire textologique, un appareil de notes et une bibliographie. Malgré l'indifférence qui a accueilli la première édition des Problèmes du comique et du rire, on peut espérer que le livre sera éventuellement traduit en français et en d'autres langues et qu'il deviendra une référence incontournable dans les études sur le comique, l'humour et le rire. L'ouvrage manque de fini et est moins percutant que le livre de Bakhtine sur Rabelais ou le Mot d'esprit et ses rapports avec l'inconscient de Freud. Néanmoins, Propp y développe une conception tout à fait originale du rire qui tranche nettement avec celles exprimées dans la plupart des études contemporaines sur le sujet. Le fait que ce livre se soit avéré le testament intellectuel d'un grand savant ne peut que le rendre encore plus précieux à nos yeux.

"Il y a une certaine manière de Propp, écrit Lise GruelApert, que l'on peut définir comme l'alliance d'une méthode inductive strictement rigoureuse à la mise en haleine, à la prise en partie de l'intérêt du lecteur" (Gruel-Apert 7). Cette observation décrit bien la façon dont procède l'auteur des Problèmes du comique et du rire. Selon Propp, toutes les études existantes sur le sujet partagent le même défaut majeur: elles ne reposent pas sur une analyse rigoureuse de faits empiriques et sont donc "totalement et horriblement abstraites"

(Propp 1999, 5). [ $\underline{2}$ ] Conséquemment, les définitions du rire qu'on y trouve sont beaucoup trop étroites et cessent d'être opératoires dès qu'on les confronte à des exemples concrets. Il est donc nécessaire de procéder de façon inductive et de tenir compte de "tout ce qui provoque un rire ou un sourire, tout ce qui est lié de près ou de loin au domaine du comique" (ㄱ). Seule une étude rigoureuse et objective de ces faits peut nous permettre de mettre à jour "l'essence du comique." Propp semble ainsi délimiter un territoire immense, quasi insondable. Il se permet toutefois d'importantes restrictions. Premièrement, son corpus comprend surtout des oeuvres 
littéraires, même s'il se réfère parfois à des contes populaires et à d'autres phénomènes non esthétiques. Deuxièmement, il se restreint à la littérature moderne (du dix-huitième au vingtième siècle), principalement à la littérature moderne russe (Gogol, SaltykovChtchédrine, Tchekhov, Ilf et Petrov, etc.).

Troisièmement, dans la première partie de l'ouvrage, il ne considère qu'un seul type de rire, le rire "moqueur" (nasmeshlivyi smekh), qui serait le type de rire dominant dans la littérature moderne. La tâche que se propose d'accomplir Propp est la suivante: pour définir l'essence du comique et la finalité du rire (moqueur), il doit, en premier lieu, classifier les procédés qui ont pour fonction, dans une oeuvre littéraire, d'exposer le risible dans les pensées, l'apparence ou les actions d'un personnage. Les procédés examinés sont au nombre de quatorze: l'accentuation des propriétés corporelles d'un être, la ressemblance, la contradiction, l'animalisation de l'humain, la chosification de l'humain, la dérision des professions, la parodie, l'exagération, la négation de la volonté, la duperie, l'alogisme, le mensonge, le comique verbal (calembours, paradoxes, mots d'esprit), le comique de caractère et le quiproquo. En examinant chaque procédé, Propp conclut que celui-ci provoque le rire uniquement lorsqu'il dévoile, de façon subite, l'existence de défauts qui, à l'origine, étaient tout à fait imperceptibles. Ainsi, l'animalisation ou la chosification d'un personnage ne sont pas nécessairement comiques dans toutes les situations; ces procédés ne suscitent le rire que lorsqu'ils révèlent subitement l'imperfection intérieure du personnage. Le comique serait donc une opération de dévoilement qui a pour but de d'exposer et châtier les vices cachés des êtres humains.

En s'appuyant sur la partie analytique de son étude, Propp propose la définition suivante du rire: "[...] nous rions lorsque dans notre conscience, les qualités positives d'une personne sont camouflées par la révélation de défauts qui apparaissent subitement à travers le voile d'apparences externes, physiques" ( $\underline{\mathbf{1 7 5}})$. Cette définition recoupe d'autres conceptions célèbres du rire. Comme le remarque Charles Mauron, Bergson et Freud "font naître le rire d'un rapport entre deux représentations; l'un des termes du rapport est tenu supérieur à l'autre et c'est lui que le rieur attendait; [...] mais à la place du premier terme, c'est le second, l'inférieur qui arrive [...]" (Mauron 146). On observe un mécanisme semblable dans la définition de Propp. Le rire naît, chez Bergson, de l'opposition entre le "vivant" et le "mécanique"; chez Freud, de l'opposition entre un contenu "adulte" et un contenu "infantile"; chez Propp, de l'opposition entre les qualités spirituelles d'un être et 
ses défauts. Propp reprend une autre idée qui est commune à beaucoup d'autres théories en concevant le rire comme un "châtiment," une "brimade sociale," dont la fonction est de condamner des éléments qui sont jugés nuisibles à la société humaine: "En voyant que le mal a été démasqué, écrit-il, et, par le fait même, qu'il subit raillerie et châtiment, nous en éprouvons satisfaction et plaisir" (Propp 1999, 182). Le rieur serait donc, comme chez Hobbes, [ $\underline{\mathbf{3}}$ ] dans une position de supériorité par rapport à l'objet de la moquerie; celui qui rit ne peut faire autrement que de se comparer à la personne visée par le rire et de constater, avec bonheur, qu'il est dépourvu des défauts dévoilés par l'opération comique. Lorsque le rire se retourne contre le rieur, comme dans la scène finale du Révizor de Gogol, lorsque le gouverneur humilié invective les spectateurs: "De quoi riez-vous?... C'est de vous-mêmes que vous riez!", alors, conclut Propp, "l'effet comique s'estompe aussitôt" (182). On ne peut rire que des défauts d'autrui.

Propp semble donc réduire tous les phénomènes comiques à la seule moquerie. Il admet certes l'existence d'autres types de rire, auxquels il consacre la deuxième partie de l'ouvrage (beaucoup plus brève que la première partie): le rire gentil, le rire méchant, le rire cynique, le rire joyeux, le rire rituel et le rire déréglé. Mais il écarte certains types comme des phénomènes extra-littéraires (rires joyeux, rituel et déréglé) ou comme des formes perverties qui ne provoquent aucun effet comique (rires méchant et cynique). Propp se demande s'il n'y aurait pas, en fait, "deux grands domaines ou types de rire. Un qui contient une moquerie, l'autre qui n'en contient pas" (151). Le rire "gentil" ne serait qu'une forme adoucie, pacifiée du rire moqueur. Dans la conclusion, Propp reprend cette idée et précise sa pensée: les deux principaux types de rire sont "le rire moqueur et le rire joyeux" (181). Le rire joyeux, qui "représente une réaction physiologique à une joie de vivre extrême" (183), ne peut être provoqué par une oeuvre artistique ou littéraire. C'est une réaction spontanée "qui surgit sans motif ou pour des raisons insignifiantes" (161). Si on suit le raisonnement de Propp, il apparaît logique de conclure que tout texte qui suscite un rire appartient nécessairement, à un degré ou à un autre, au vaste domaine de la moquerie. En quittant ce domaine, on quitte par le fait même le domaine du comique. 
Pour saisir l'originalité et le sens polémique des idées de Propp, il est important de situer son ouvrage dans le contexte des études littéraires soviétiques. La question du rire a souvent été à l'ordre du jour dans les milieux littéraires en URSS et a parfois été à l'origine de débats houleux, notamment à la fin des années 1920 et au milieu des années 1960 (lorsque Propp entreprend la rédaction des Problèmes du comique et du rire). À travers ces multiples débats, la conception officielle, "orthodoxe" du rire reste sensiblement la même: la seule forme de rire acceptable est la satire qui est utilisée comme un instrument au service de la lutte des classes; le rire doit avoir une signification sociale, politique et idéologique et doit contribuer à l'édification de l'état socialiste en visant soit des ennemis extérieurs (le capitalisme, le fascisme), soit des ennemis intérieurs (les classes défaites, les "survivances" du passé, les saboteurs). [ $\underline{4}$ ] Propp, qui a toujours évité durant sa carrière de savant d'être mêlé à des conflits idéologiques, reste très prudent lorsqu'il aborde ce terrain miné. Dans certains passages, il cite les "classiques" (Marx, Engels, Lénine) et semble d'accord avec l'idée que la satire peut remplir une fonction politique à des époques cruciales de l'histoire (par exemple, après la Révolution d'octobre et durant la guerre). Mais la conception du comique qu'il développe dans l'ensemble de l'ouvrage va à l'encontre des dogmes de la critique officielle. Selon Propp, les défauts que dévoile l'opération comique sont principalement - sinon exclusivement - de "petits défauts" (melkie dedostatki), des défauts "d'ordre spirituel" (dukhovnogo poriadka) qui concernent "des principes moraux, des questions de volonté ou des opérations mentales" (175). Ainsi, la lâcheté dans la vie quotidienne peut être une source de rire, mais pas la lâcheté à la guerre (132). Propp accorde une attention particulière à la dimension spécifiquement morale du comique. Le sentiment de supériorité que suscite le comique "a un caractère profondément moral" (182). Le rire moqueur est l'expression "d'une justice triomphante", le signe d'une "victoire morale" (183). La jubilation du rieur est fondée sur la reconnaissance de la nécessité de principes moraux "que l'autre ne possède pas, mais que moi je possède" (182). C'est en s'efforçant d'enrayer "toutes les formes de vilenie morale" (218) que l'auteur comique peut jouer un rôle social et contribuer à l'édification d'un monde meilleur. 
Selon Propp, cette finalité morale caractérise toutes les oeuvres comiques et constitue l'essence même du comique. Il est donc absurde d'opposer la satire, qui serait un genre "élevé," ayant une signification idéologique, socio-politique, et l'humour, qui serait un genre "bas," ne traitant que de problèmes particuliers et insignifiants. Une telle opposition, soutient Propp, découle d'une confusion terminologique. La distinction entre la satire et l'humour est un non-sens, puisque "dans les deux cas, les procédés comiques sont identiques" (188). Ainsi, qu'un auteur ait eu l'intention de corriger les moeurs ou de simplement divertir son public, que son oeuvre soit catégorisée comme de la satire ou de l'humour, peu importe: dans un cas comme dans l'autre, si son texte fait rire, il accomplit une opération comique qui a pour but de dévoiler des défauts cachés et d'aiguiser le sens moral du public. Le contenu politique et idéologique de la satire excède nécessairement sa finalité comique. Si on veut exclure des oeuvres qui ont été écrites uniquement pour le divertissement, conclut Propp, il faudrait alors condamner de nombreuses comédies de Shakespeare et presque toutes les comédies du répertoire classique (192).

Les idées de Propp sur le rire risquent peut-être de paraître inactuelles, pour ne pas dire désuètes, aux lecteurs contemporains. Le livre de Bakhtine qui valorise la force subversive du rire carnavalesque et la théorie de Freud qui traite le rire comme le signe de pulsions inconscientes sont beaucoup plus adaptés aux goûts du jour, particulièrement dans les milieux universitaires. Paradoxalement, c'est peut-être par son inactualité que la notion proppienne de "comique moral" peut ouvrir des perspectives nouvelles et servir de source d'inspiration aux chercheurs qui s'intéressent à la question du rire. Même si on prétend souvent que l'humour, pour reprend l'expression de Kundera, est un "territoire où le jugement moral est suspendu" (Kundera 16), même si cette idée tend à s'imposer comme un dogme, on entend parfois des voix solitaires qui affirment le contraire. Dans un entretien récent, par exemple, Daniel Lemire, un des plus grands humoristes québécois - et de l'avis de plusieurs, un des plus drôles -, affirme que "sans éthique, l'humour n'a pas de sens" (cité par Baillargeon). "Je pense que l'humour, explique-t-il, doit servir à faire rire, 
oui, mais aussi à mettre en relief certaines tendances, certains comportements, certaines idées" (idem). De tels propos font écho à la thèse défendue par l'auteur des Problèmes du comique et du rire.

On doit également souligner la valeur indiscutable de la partie analytique de l'ouvrage. Même s'il existe déjà une quantité impressionnante d'études sur le comique et l'humour en langue française, il manque encore une étude rigoureuse des procédés comiques dans le discours littéraires. Plusieurs ouvrages, tels l'Écriture comique de Jean Sareil et le Comique de Jean Emelina, ont déjà comblé partiellement cette lacune, mais ils ne rendent pas compte de tous les mécanismes narratifs, rhétoriques et discursifs dont le but est de provoquer le rire. La classification proposée par Propp n'est pas plus exhaustive, mais elle a le mérite de compléter ou corriger les grilles des autres chercheurs.

Une autre qualité de l'ouvrage est sa fibre personnelle, subjective. Malgré les prétentions de Propp à une vérité objective et empirique, il se permet souvent d'émettre des jugements de valeur qui ne sont pas dépourvus d'une charge émotive. Par exemple, lorsqu'il affirme, au sujet de Gogol, qu'il est "le plus grand auteur humoristique et satirique de tous les temps, laissant loin derrière lui tous les autres maîtres du genre, russes et non russes" (Propp 1999, 7). Ou encore lorsqu'il condamne la raillerie cynique comme une forme de perversion qui détourne la finalité morale du comique à des fins haineuses et misanthropiques (158-161). En lisant les Problèmes du comique et du rire, il faut garder à l'esprit cette formule d'Ionesco: "Où il n'y a pas d'humour, il n'y a pas d'humanité; où il n'y pas d'humour, il y a le camp de concentration" (cité par Elgozy 22). Les pages les plus lumineuses du livre sont celles où Propp se porte à la défense du rire comme l'expression des meilleurs sentiments et aspirations de l'espèce humaine (notamment le chapitre 3 "Ceux qui rient et ceux qui ne rient pas"). Le sens de l'humour, affirme-t-il, caractérise non seulement les gens les plus talentueux, mais aussi tous les gens "normaux et vivants"; tandis que l'absence de sens d'humour peut être interprétée comme un signe "d'idiotie et d'insensibilité," et même "de perversité" (24-25). Propp a écrit ces lignes dans le contexte morose de l'Union Soviétique de la fin des années 1960. On peut croire qu'avant de mourir, le grand savant soviétique tenait à défendre un domaine de la culture qu'il considérait comme une valeur spirituelle essentielle, mais qui était devenu suspect aux yeux des idéologues du parti. Propp a peut-être voulu quitter le monde en 
riant, en préservant, dans ses écrits, une dernière étincelle d'humanité.

\section{Textes cités}

Bakhtine, Mikhaïl. L'Oeuvre de François Rabelais et la culture comique populaire au Moyen Âge et sous la Renaissance. Tr. Andrée Robel. Paris: Gallimard, coll. Tel, 1982.

Baillargeon, Stéphane. "Daniel Lemire: l'humoriste de la responsabilité." Le Devoir. 31 mars 2000 http:// www.ledevoir.com/pop/2000a/lemi310300.html.

Bergson, Henri. Le Rire. Essai sur la signification du comique. Paris: P.U.F., coll. Quadrige, 1993.

Blondel, Éric. Le Risible et le dérisoire. Paris: P.U.F., coll. Perspectives critiques, 1988.

Bremond, Claude. Logique du récit. Paris: Seuil, coll. Poétique, 1973.

Elgozy, Georges. De l'humour. Paris: Denoël, 1979.

Emelina, Jean. Le Comique. Essai d'interprétation générale. Paris: Sedes, 1991.

Freud, Sigmund. Le Mot d'esprit et sa relation à l'inconscient. Tr. Denis Mercier. Paris: Gallimard, coll. Folio/Essais, 1992.

Gruel-Apert, Lise. "Introduction." Vladimir Propp. Les Racines historiques du conte merveilleux. Paris:

Gallimard, 1983: 3-8.

Kundera, Milan. Les Testaments trahis. Paris: Gallimard, 1993.

Mauron, Charles. Psychocritique du genre comique.

Paris: José Corti, 1964.

Ozmitel', Evgenii. Sovetskaia satira. Moskva-Leningrad: Prosveshchenie, 1964.

Propp, Vladimir. Morphologie du conte. Tr. Marguerite Derrida. Paris: Seuil, coll. Poétique, 1970.

-. Problemy komizma i smekha. Moskva: Labirint, 1999. 
Sareil, Jean. L'Écriture comique. Paris: P.U.F., 1984.

\section{NOTES}

1. Voir, par exemple, "L'héritage de Propp" dans la Logique du récit de Claude Bremond (9-47).

2. Je traduis toutes les citations du russe.

3. "[L]a passion du rire procède d'une représentation soudaine de notre avantage et de notre supériorité personnelle. [...] [L]a passion du rire n'est autre qu'une gloire [vanité] soudaine née de la perception d'une supériorité de notre part [...]" (cité par Blondel 127).

4. Pour plus de renseignements sur la conception soviétique de la satire, on peut consulter, en russe, le chapitre "Voprosy teorri i istorii sovetskoi satiry v kritike i literaturovedenii" dans l'ouvrage de Evgenii Ozmitel', Sovetskaia satira (5-49).

Accueil Surfaces | Table des matières | Recherche $\underline{\text { Surfaces Home Page | Table of Contents | Search }}$

PUM | Livres | Revues | Publications électroniques | Vente et distribution 